\title{
Transition metal-free oxidation of benzylic alcohols to carbonyl compounds by hydrogen peroxide in the presence of acidic silica gel
}

\author{
Hossein Ghafuri*
}

Catalysts and Organic Synthesis Research Laboratory, Department of Chemistry, Iran University of Science and Technology, Tehran, Iran

\section{H R O N I C L E}

Article history:

Received May 6, 2014

Received in revised form

October 02, 2014

Accepted 8 November 2014

Available online

8 November 2014

Keywords:

Oxidation

Carbonyl compounds

Hydrogen peroxide

Acidic silica gel \begin{abstract}
A B S T R A C T
Oxidation of alcohols to carbonyl compounds has become an important issue in the process industry as well as many other applications. In this method, various benzylic alcohols were successfully converted to corresponding aldehydes and ketones under transition metal-free condition using hydrogen peroxide in the presence of some amount of catalytic acidic silica gel. Silica gel is inexpensive and available. One of the most important features of this method is its short reaction time.
\end{abstract}

\section{Introduction}

Oxidation of alcohols to carbonyl compounds is fundamental to organic chemistry, and is currently attracting a lot of interest. Oxidation of alcohols to carbonyl compounds has established itself as an important application in the chemical process industry. Carbonyl compounds such as aldehydes and ketenes are used in the synthesis of many organic compounds, vitamins, complex ligands as well as medicine ${ }^{1}$. Benzaldehyde is a valuable chemical substance, and has widespread applications in chemical industries ${ }^{2}$. However, most of these reagents of transition metal complexes such as chromium and cobalt are often either toxic or hard to prepare.

Due to its importance, several papers in the field have been devoted to this topic. In some of these, a great number of oxidizing agents have been identified for the oxidation of benzyl alcohols ${ }^{3-}$ ${ }^{17}$. Nevertheless, some of the reported reagents and catalysts suffer from disadvantages such as lack of availability, difficult work-up, long reaction time, cost of metal catalysts, as well as toxicity and high cost of the reagents. Thus, milder and more selective reagents and transition metal-free eco-friendly

\footnotetext{
* Corresponding author. Fax: +98-21-7322 7703

E-mail address: ghafuri@iust.ac.ir (H. Ghafuri) 
systems are still in great need. Recently, the use of solid supported reagents has received considerable attention in organic synthesis because of its ease of handling, enhanced reaction time, greater selectivity power, simple workup, and recoverability of catalysts ${ }^{18}$. Among the various heterogeneous catalysts, silica gel is advantageous not only for its low cost but also for its ease of preparation, especially when it is impregnated with hydrochloric acid. Since the reaction is heterogeneous in nature, the catalyst can conveniently be separated through simple filtration.

Over the past few years, the importance of hydrogen peroxide and its derivatives as oxidizing agents has grown considerably. From an environmental viewpoint, in contrast to other oxidizing agents, hydrogen peroxide is the most attractive one, and- as water is the only theoretical byproduct in this process- is an ideal waste-avoiding oxidant, hence its attractiveness as a good oxidant, thanks to its solubility in water as well as its numerous available organic solvents. Moreover, aqueous hydrogen peroxide solution lends itself safely to storage, operation as well as transportation, and is relatively cheap and widely available in the market ${ }^{19}$. Recently Sharma et al. reported oxidation of alcohols to carbonyl compounds using aqueous $\mathrm{HBr}$ and hydrogen peroxide in acetonitrile under the reflux condition ${ }^{20}$. Hong Yun Guo has recently reported using $\mathrm{H}_{2} \mathrm{O}_{2} / \mathrm{HCl}$ for the synthesis of substituted benzothiazoles ${ }^{21}$. In 1977, Hurst made a detailed investigation of kinetic parameters for oxidation of hypochlorous acid by hydrogen peroxide and yield formation of excited oxygen (or singlet oxygen, eq. 1$)^{22}$.

$\mathrm{H}_{2} \mathrm{O}_{2}+\mathrm{HOCl} \longrightarrow{ }^{1} \mathrm{O}_{2}+\mathrm{H}_{2} \mathrm{O}+\mathrm{H}^{+}+\mathrm{Cl}^{-}$

Photocatalytic oxidation of benzyl alcohol to benzaldehyde by oxygen is a product of $\mathrm{H}_{2} \mathrm{O}_{2}{ }^{23}$ (Eq. (2)).

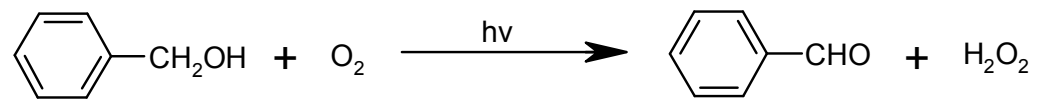

\section{Results and discussion}

As part of our research on the development of supported reagents and catalysts for the oxidation of alcohols, we have recently developed a heterogeneous catalytic method based on sodium nitrite in the presence of acidic silica gel for effective aromatization of Hantzsch 1,4-dihydropyridines ${ }^{24}$. Here we describe this catalyst (acidic silica gel) for effective oxidation of benzyl alcohols to corresponding aldehydes and ketones using hydrogen peroxide as an oxidant (Scheme 1).

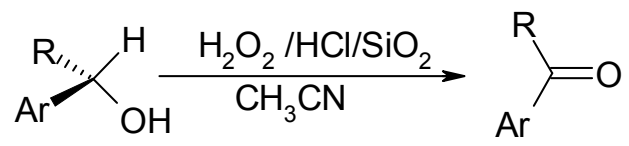

Scheme 1. Oxidation of benzyl alcohols

In this paper, acidic silica gel along with hydrogen peroxide is found to be a simple metal free oxidation reagent. Silica gel has such advantages as low cost and ease of preparation, and since the reaction is heterogeneous in nature, the catalyst can conveniently be separated with simple filtration. Due to its solubility in water, hydrogen peroxide is a very convenient oxidizing agent. The reaction yield is high, with water as the only by-product, which is environmentally friendly. The catalyst (acidic silica gel) is stable, and can easily be prepared by adding silica gel to aqueous hydrochloric acid and evaporating the solvent. Different types of alcohols were subjected to oxidation with hydrogen peroxide using acidic silica gel as catalyst. The oxidation of alcohols is carried out in acetonitrile as solvent, and the workup simply requires extraction and separation of the heterogeneous 
catalyst through filtration. Removal of solvent followed by purification of products aldehydes and ketones in high yields. The respective reaction times and yields are given in Table 1.

Table 1. Oxidation of alcohols by hydrogen peroxide in the presence of acidic silica gel

Entry

As shown in Table 1, benzylic alcohols are oxidized to corresponding carbonyl compounds in good yields, but the yields for secondary alcohols (Entry 12,13) are lower using this condition. Some experiments were performed in order to show the utility of the methodology. The oxidation of benzylic alcohols to the corresponding carbonyl compounds was found to be very slow at room temperature, while it could be carried out more efficiently in refluxing acetonitrile. A reaction condition is optimized not only in terms of solvent and temperature, but also with respect to the amount of acidic silica gel and oxidant. In select solvents such as $\mathrm{CH}_{2} \mathrm{Cl}_{2}, \mathrm{THF}$, DMF and water low yields were obtained. $\mathrm{CH}_{3} \mathrm{CN}$ was found to be the most suitable solvent for this transformation with the shortest reaction time and good yields (Table 2).

The optimum temperature was $80^{\circ} \mathrm{C}$, and the optimum amount of $\mathrm{SiO}_{2}-\mathrm{HCl}$ was 0.5 gram for 1 mmole alcohols. No Reactions happened at room temperature or without $\mathrm{SiO}_{2}-\mathrm{HCl}$; thus, only the initial materials were recovered. The real catalyst was found to be $\mathrm{HCl}$, where silica gel served only as a shuttle for delivering $\mathrm{HCl}$ in solution. Furthermore, when the reaction runs under an inert atmosphere of argon without atmospheric oxygen, it renders good yields of products, which repel 
oxidation with oxygen. Finally, oxidation of benzylic alcohols over acidic silica gel with $\mathrm{O}_{2}$ under the same reaction condition resulted only in the initial materials. After the activation the catalyst can be used.

Table 2. Optimization of reaction condition

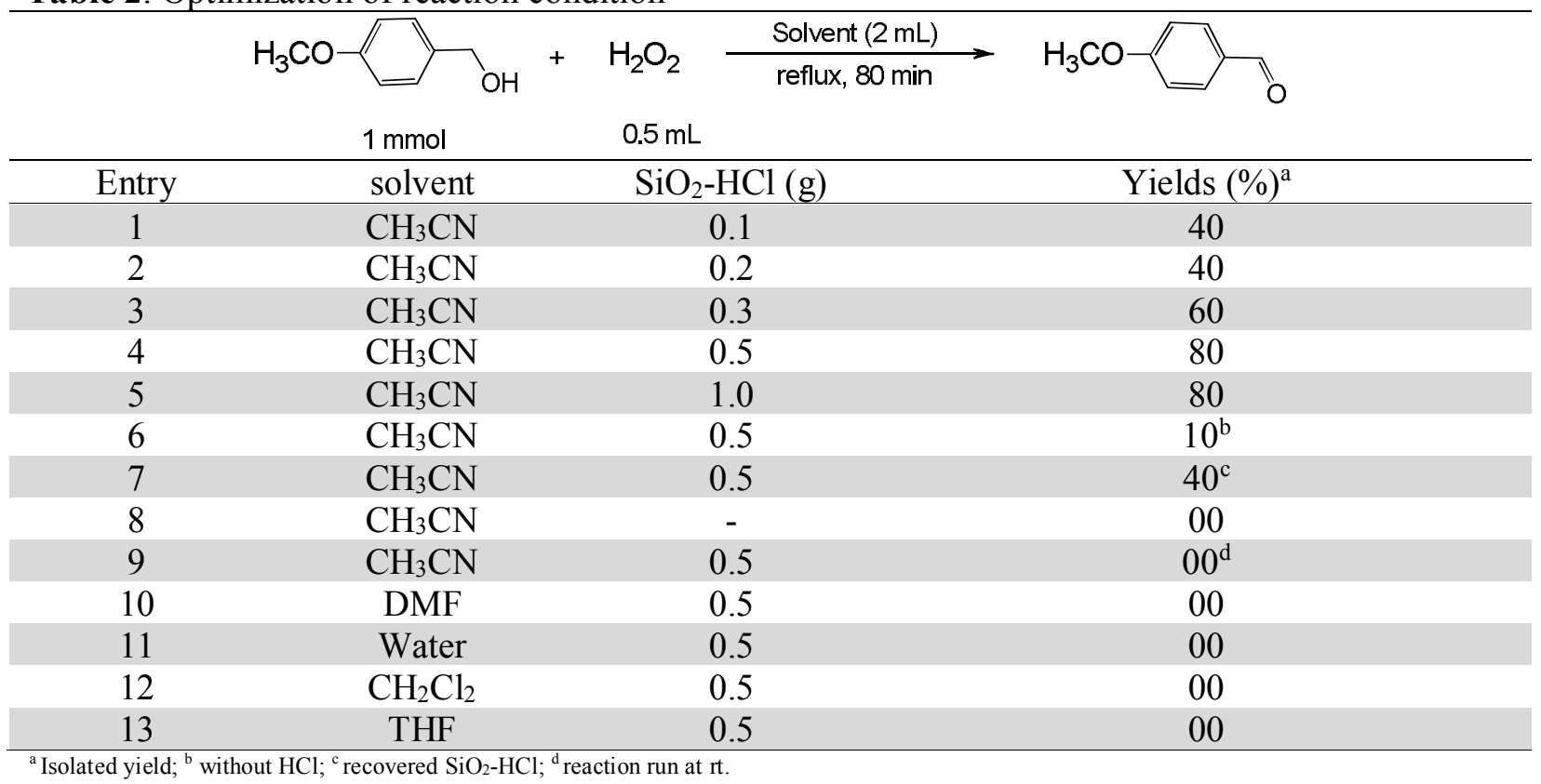

Accordingly, the mechanism of alcohol oxidation to carbonyl compounds through hydrogen peroxide acidic silica gel is proposed in scheme 2 .

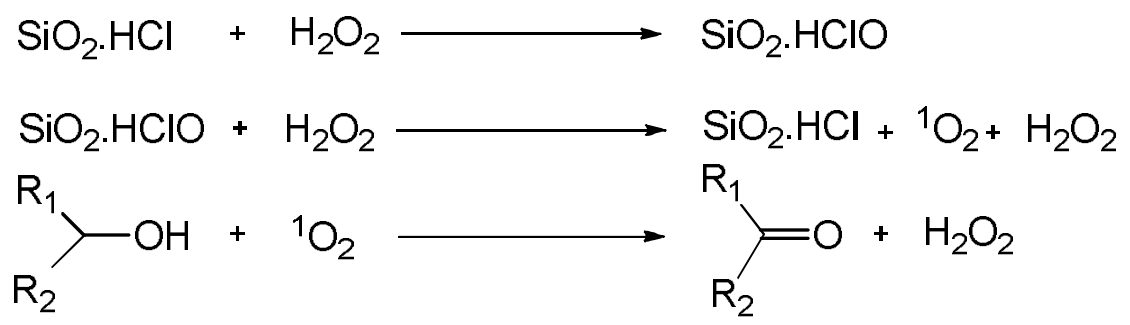

Scheme 2. Suggested mechanism

\section{Conclusion}

As demonstrated above, in this paper, we have successfully extended the application of hydrogen peroxide and acidic silica gel for the oxidation process of alcohols. Due to its simplicity of performance, metal-free oxidation, good yields, and the wide range of benzylic alcohols that can be converted into their corresponding aldehydes and ketones, this method offers a lot of advantages over its rivals, and yet the silica gel can ultimately be recycled after activation.

\section{Experimental}

The products were characterized by comparing their physical and spectral data against those obtained from authentic samples. All yields refer to isolated products. IR and NMR spectra were recorded on Perkin Elmer 781, and Broker DPX500. The progress of reactions was monitored by TLC. 


\subsection{Preparation of the catalyst: Acidic silica gel}

Chromatographic Silica gel (1 g, 70-230 mesh) was added to $1 \mathrm{~mL}$ conc. $\mathrm{HCl}$ and stirred at room temperature for $10 \mathrm{~min}$. The mixture was evaporated and dried in vacuum [20].

\subsection{Typical Experimental Procedure}

To a stirred solution of $1 \mathrm{mmol}$ alcohols in $2 \mathrm{~mL}$ acetonitrile, $0.5 \mathrm{~g}$ acidic silica gel was added. Then $0.5 \mathrm{~mL}$ of $\mathrm{H}_{2} \mathrm{O}_{2} 30 \%$ was gradually added to the same solution over a period of $5 \mathrm{~min}$. The resulting mixture was stirred under reflux of acetonitrile for the time indicated in Table 1. After completion of the reaction (TLC, ethyl acetate/petroleum ether; $1: 4$ ), the heterogeneous mixture was filtered and thoroughly washed with the acetonitrile $(2 \times 10 \mathrm{ml})$. The solvent was evaporated, and the product was purified through short column chromatography over silica gel.

\section{Acknowledgment}

I am deeply grateful to the Research Council at the University of Science and Technology (IUST) of Iran for their financial support of this work.

\section{References}

1 Hudlicky T., Luna H., Price J. D., and Rulin F. (1990) Microbial oxidation of chloroaromatics in the enantiodivergent synthesis of pyrrolizidine alkaloids Trihydroxyheliotridanes.J.Org. Chem.,55(15), 4683-4687.

2 Chaudhari M. P., Sawant S. B. (2005). Kinetics of heterogeneous oxidation of benzyl alcohol with hydrogen peroxide.Chem. Eng. J., 106(39), 111-118.

3 Hashemi M. M., and Beni Y. A. (2000) Oxidation of alcohols and primary aliphatic amines to carbonyl compounds using sodium hypochlorite adsorbed on montmorillonite K10. J. Chem. Res.,5, 224-225.

4 Hashemi M. M., Khalili B., and Eftekhari-Sis B. (2005) Oxidation of benzylic alcohols to carbonyl compounds with hydrogen peroxide catalysed by manganeses chloride supported onontmorillonite k10. J. Chem. Res., 8, 484-485.

5 Hashemi M. M., Rahimi A., Karimi-Jaberi Z., and Ahmadibeni Y. (2005) A mild and efficient oxidation of benzylic alcohols without solvent using iodic acid supported on wet montmorillonite K10 or silica gel under microwave irradiation. Acta Chim. Slov.,52, 86-87.

6 Hashmi M. M., Ezabadi A., and Karimi-Jaberi Z. (2005) Oxidation of aromatic alcohols to carbonyl compounds with oxygen catalyzed by iron (iii) chloride supported on kieselguhr. Lett. Org. Chem., 2(6), 559-560.

7 Firouzabadi H., Fakoorpour M., and Hazarkhani H. (2001) Highly selective oxidation of primary and secondary benzylic alcohols by $\mathrm{KMnO}_{4} / \mathrm{ZrOCl}_{2} .8 \mathrm{H}_{2} \mathrm{O}$ in diethyl ether. Synth.Common., 31(24), 3859-3862.

8 Varma S. R., Saini K. R.(1998) Selective oxidations using alumina-supported iodobenzene diacetate under solvent-free conditions. J. Chem. Res., 3, 120-121.

9 Xu L., and Trudell M. L. (2003) A mild and efficient oxidation of alcohols to aldehydes and ketones with periodic acid catalyzed by chromium(iii)acetylacetonate.Tetrahedron Lett.,44(12)., 2553-2555.

10 Lou J.-D., and Xu Z.-N. (2002) Selective solvent-free oxidation of alcohols with potassium dichromate. Tetrahedron Lett.,43(49), 8843-8844.

11 Annunziatini C., Gerini M. F., Lanzalunga O., and Lucarini M. (2004) Aerobic oxidation of benzyl alcohols catalyzed by aryl substituted n-hydroxyphthalimides. Possible involvement of a chargetransfer complex..J.Org. Chem., 69(10), 3431-3438. 
12 Lingaiah N., Reddy K. M., Babu N. S., Rao K. N., Suryanarayana I., and Prasad P. S. S. (2006) Aerobic selective oxidation of benzyl alcohol over vanadium substituted ammonium salt of 12molybdophosphoric acid. Catal. Commun.,7(4), 245-250.

13 Salavati-Niasari M. (2006) Host (nanocavity of zeolite-y)-guest (tetraaza[14]annulene copper(ii) complexes) nanocomposite materials: Synthesis, characterization and liquid phase oxidation of benzyl alcohol.J. Mol. Catal. A: Chem.,245(1-2), 192-199.

14 Jain S. L., and Sain B. (2006) Efficient transition-metal-free oxidation of benzylic and secondary alcohols to the carbonyl compounds using an n-bromosuccinimide.Synth. Common., 36(10), 14591462.

15 Zhang S., Xu L., and Trudell M. L. (2005) Selective oxidation of benzylic alcohols and tbdms ethers to carbonyl compounds with $\mathrm{CrO}_{3}-\mathrm{H}_{5} \mathrm{IO}_{6}$. , Synthesis, 36(46), 1757-1760.

16 Chhikara B. S., Chandra R., and Tandon V. (2005) A versatile method for the hydrogen peroxide oxidation of alcohols using ptc condition in tert-butanol. Synlett.,5,872-874.

17 Özgün B., and Değirmenbaş1 N. (2004) Oxidation of substituted benzyl alcohols by quinoxalinium dichromate a kinetic study. Monatsh. Chem., 135(5), 483-491.

18 Dörwald F.Z. (2002) Organic synthesis on solid phase: supports, linkers, reactions. Wiley-VCH, Weinheim.

19 Jones C.W. (1999) Applications of hydrogen peroxide and derivatives. Royal Society of Chemistry, Cambridge.

20 Sharma V. B., Jain S. L., and Sain B. (2005) A new and efficient transition metal-free oxidation of secondary alcohols to ketones using aqueous $\mathrm{HBr}$ and $\mathrm{H}_{2} \mathrm{O}_{2}$. Synlett., 1,173-175.

21 Guo Y. H., Li C.J., Shang L.Y. (2009) Asimple and efficient synthesis of 2-substituted benzothiazoles catalyzed by $\mathrm{H}_{2} \mathrm{O}_{2} / \mathrm{HCl}$. Chin. Chem. Lett., 20, 1408-1410..

22 Held A. M., Halko D. J., and Hurst J K. (1978) Mechanisms of chlorine oxidation of hydrogen Peroxide. J. Amer. Chem. Soc., 100, 5732.

23 Ohkubo K., Suga K and Fukuzumi S. (2006) Solvent-free selectivephotocatalytic oxidation of benzyl alcohol to benzaldehyde by molecular oxygen using 9-phenyl-10-mhylacridinium.Chem. Commune., 2018-2020.

24 Hashemi M. M., Ghafuri H., and Karimi-Jaberi Z. (2006) Room temperature catalytic aromatization of hantzsch 1,4-dihydropyridines by sodium nitrite in the presence of acidic silica gel. Monatsh. Chem., 137(2), 197-200. 